\title{
ATRIBUTOS FUNCIONAIS DE ESPÉCIES ARBÓREAS EM UM FRAGMENTO DE FLORESTA OMBRÓFILA MISTA EM LAGES - SC
}

\author{
FUNCTIONAL TRAITS OF TREE SPECIES IN A FRAGMENT OF ARAUCARIA FOREST IN \\ LAGES, SANTA CATARINA STATE
}

\begin{abstract}
Francieli de Fátima Missio ${ }^{1}$ Ana Carolina da Silva ${ }^{2}$ Pedro Higuchi ${ }^{3}$ Solon Jonas Longhi ${ }^{4}$ Martha Andréia Brand ${ }^{5}$ Polliana D'Angelo Rios ${ }^{6}$ Angélica Dalla Rosa ${ }^{7}$ Fernando Buzzi Junior ${ }^{8}$ Marco Antônio Bento ${ }^{9}$ Didiane Ana Gonçalves ${ }^{10}$ Rodineli Loebens ${ }^{11}$ Francieli Pscheidt ${ }^{12}$
\end{abstract}

\section{RESUMO}

O presente estudo teve como objetivo descrever atributos funcionais de espécies arbóreas em um fragmento de Floresta Ombrófila Mista em Lages - SC, com o propósito de agrupá-las funcionalmente. Para cada uma das 20 espécies mais abundantes na área de estudo foi amostrado um total de 10 indivíduos e determinada a média da densidade da madeira e o tamanho médio das folhas. Dessas espécies, também foi descrito o regime de renovação foliar, a síndrome de dispersão de propágulos e a altura máxima no fragmento. Os resultados de altura máxima e de densidade da madeira sugerem a existência de um trade-off entre $\mathrm{o}$ investimento de recursos para o crescimento em altura, garantindo o acesso à maior disponibilidade lumínica, ou em densidade da madeira, permitindo maior capacidade de resistência e sobrevivência em um ambiente sombreado de sub-bosque. As gimnospermas Araucaria angustifolia e Podocarpus lambertii apresentaram as folhas de menor tamanho, enquanto Cupania vernalis, Jacaranda puberula e Matayba elaeagnoides, todas de folhas compostas, apresentam as maiores folhas. Quatro grupos funcionais se destacaram: 1) perenes de folhas grandes, 2) perenes de folhas pequenas, 3) decíduas zoocóricas e 4) decíduas não zoocóricas. Os resultados demonstraram que as espécies mais abundantes do fragmento de Floresta Ombrófila Mista estudado apresentam variações dos atributos funcionais avaliados, sugerindo

1 Engenheira Florestal, MSc., Departamento de Engenharia Florestal, Universidade do Estado de Santa Catarina. Av. Luiz de Camões, 2090, CEP 88520-000, Lages (SC), Brasil. franmissio@yahoo.com.br

2 Engenheira Florestal, Dra ${ }^{\text {. }}$, Professora do Departamento de Engenharia Florestal, Universidade do Estado de Santa Catarina. Av. Luiz de Camões, 2090, CEP 88520-000, Lages (SC), Brasil. carol_sil4@yahoo.com.br

3 Engenheiro Florestal, Dr., Professor do Departamento de Engenharia Florestal, Universidade do Estado de Santa Catarina. Av. Luiz de Camões, 2090, CEP 88520-000, Lages (SC), Brasil. higuchip@gmail.com

4 Engenheiro Florestal, Dr., Professor do Departamento de Ciências Florestais, Universidade Federal de Santa Maria. Av. Roraima, 1000, CEP 97105-900, Santa Maria (RS), Brasil. solon.longhi@gmail.com

5 Engenheira Florestal, Dra ${ }^{\mathrm{a}}$, Professora do Departamento de Engenharia Florestal, Universidade do Estado de Santa Catarina. Av. Luiz de Camões, 2090, CEP 88520-000, Lages (SC), Brasil. martha.brand@udesc.br

6 Engenheira Florestal, Dra ${ }^{\mathrm{a}}$, Professora do Departamento de Engenharia Florestal, Universidade do Estado de Santa Catarina. Av. Luiz de Camões, 2090, CEP 88520-000, Lages (SC), Brasil. polliana.rios@udesc.br

7 Engenheira Florestal, Departamento de Engenharia Florestal, Universidade do Estado de Santa Catarina. Av. Luiz de Camões, 2090, CEP 88520-000, Lages (SC), Brasil. angelica.dalla.rosa@hotmail.com

8 Engenheiro Florestal, Departamento de Engenharia Florestal, Universidade do Estado de Santa Catarina. Av. Luiz de Camões, 2090, CEP 88520-000, Lages (SC), Brasil. buzzifjr@hotmail.com

9 Engenheiro Florestal, Departamento de Engenharia Florestal, Universidade do Estado de Santa Catarina. Av. Luiz de Camões, 2090, CEP 88520-000, Lages (SC), Brasil. marco_a_bento@hotmail.com

10 Engenheira Florestal, Departamento de Engenharia Florestal, Universidade do Estado de Santa Catarina. Av. Luiz de Camões, 2090, CEP 88520-000, Lages (SC), Brasil. didi_ana15@hotmail.com

11 Engenheiro Florestal, MSc., Departamento de Engenharia Florestal, Universidade do Estado de Santa Catarina. Av. Luiz de Camões, 2090, CEP 88520-000, Lages (SC), Brasil. rhodineli@hotmail.com

12 Engenheira Florestal, MSc., Departamento de Engenharia Florestal, Universidade do Estado de Santa Catarina. Av. Luiz de Camões, 2090, CEP 88520-000, Lages (SC), Brasil. francieli_pscheidt@hotmail.com

Recebido para publicação em 17/04/2014 e aceito em 19/06/2015

Ci. Fl., v. 27, n. 1, jan.-mar., 2017 
diferentes estratégias de vida.

Palavras-chave: comunidade arbórea; estratégias ecológicas; alocação de recursos.

\begin{abstract}
This study aimed to describe the functional traits of tree species in an Araucaria Forest fragment, in the municipality of Lages, SC state, in order to group them functionally. For each of the 20 most abundant species in the study area, a total of 10 individuals were sampled for the determination of the mean wood density and mean leaf size. The leaf renovation regimes, the dispersal syndrome and the maximum height in the fragment were also described for these species. The results of maximum height and wood density suggest the existence of a trade-off between investment of resources in height growth, ensuring access to some higher light availability, or in wood density, allowing for greater resilience and survival in a shaded understory environment. The gymnosperm species, Podocarpus lambertii and Araucaria angustifolia showed smaller leaves, while Cupania vernalis, Jacaranda puberula and Matayba elaeagnoides, all of compound leaves, had larger ones. Four functional groups stood out: 1) Evergreen with large leaves, 2) Evergreen with small leaves, 3) Deciduous with zoochoric syndrome, and 4) Deciduous with nonzoochoric syndrome. The results showed that the most abundant species of the studied Araucaria Forest fragment present functional trait variations, suggesting different life strategies.
\end{abstract}

Keywords: Tree species community; ecological strategies; resource allocation.

\title{
INTRODUÇÃO
}

Estudos com enfoque na preservação e manutenção das florestas se tornam cada vez mais direcionados para a relação das características morfofuncionais das espécies arbóreas e suas condições de adaptação diante da heterogeneidade ambiental existente (WALKER, 1992), permitindo, assim, um melhor entendimento sobre a organização de ecossistemas florestais. Essa abordagem busca o entendimento de como as características morfológicas e fisiológicas das espécies afetam seus respectivos desempenhos na comunidade, diante da variabilidade de recursos e condições ambientais (MATTOS et al., 2004). Nesse sentido, estudos têm demonstrado os diferentes mecanismos adaptativos que as espécies de plantas desenvolvem e a influência destes sobre a funcionalidade dos ecossistemas (e.g. CORNELISSEN et al., 2003; WEBB et al., 2010).

Segundo Violle et al. (2007), atributos funcionais são as características dos organismos que influenciam no seu desenvolvimento e que resumem as inúmeras formas que estes utilizam um determinado recurso para o seu desenvolvimento e sobrevivência. Assim, as plantas podem ser categorizadas a partir de seus atributos funcionais (LAVOREL et al., 1997), que variam de acordo com as estratégias ecológicas e as diferentes formas de aquisição dos recursos no ecossistema (WESTOBY et al., 2002). Desta forma, categorizar as espécies arbóreas de uma comunidade florestal de acordo com os atributos funcionais permite, em grande parte, agrupá-las funcionalmente, com características morfofisiológicas semelhantes (GITAY; NOBLE, 1997; PILLAR; DUARTE, 2010), uma vez que essas plantas respondem de forma similar às diferentes condições ambientais (CORNELISSEN et al., 2003). Esta abordagem possibilita um maior entendimento sobre a organização de comunidades, assim como, sobre os padrões de distribuição dessas espécies ao longo dos gradientes ambientais.

As variações nos atributos funcionais podem apresentar correlações ecológicas importantes que ocorrem na comunidade. Por exemplo, a variação da área foliar apresenta relação com as taxas de crescimento da planta e está relacionada com a capacidade de captação de luz (CORNELISSEN et al., 2003; VALLADARES; BRITES, 2004). O regime de renovação foliar apresenta-se associado à sazonalidade climática ao longo do ano e às propriedades físico-químicas do solo (GIVNISH, 1987), e está relacionado com as estratégias de utilização de nutrientes pelas plantas, representando, assim, um importante indicativo sobre taxas de crescimento, eficiência de uso de nutrientes e decomposição da serapilheira (PÉREZ-HARGUINDEGUY et al., 2013). Já o material lenhoso, relacionado à densidade da madeira, desempenha funções de suporte mecânico para a copa, responsável pela realização da fotossíntese, transporte de seiva no sistema solo-planta-atmosfera e armazenamento de água e outros recursos 
(CHAVE et al., 2009), estando, desta forma, correlacionada a várias propriedades morfológicas, fisiológicas e ecológicas (CHAVE et al., 2006) e com grande influência no sequestro de carbono (CHAVE et al., 2009). A altura máxima, por sua vez, relaciona-se com a competição por luz, influenciando a estratificação da floresta (GIEHL et al., 2007). A síndrome de dispersão está relacionada ao deslocamento dos propágulos por dispersão anemocórica, autocórica e zoocórica (VAN DER PIJL, 1982), indicando a capacidade de ocupação espacial das espécies.

Considerando a importância da análise dos atributos funcionais de espécies arbóreas para o entendimento das estratégias de desenvolvimento das mesmas em ecossistemas florestais, o presente estudo teve como objetivos: i) descrever os atributos funcionais de espécies arbóreas em um fragmento de floresta Ombrófila Mista em Lages, SC; e ii) agrupá-las funcionalmente, para fins de uma melhor compreensão sobre as estratégias de vida das mesmas e sobre o funcionamento da comunidade arbórea.

\section{MATERIAL E MÉTODOS}

O estudo foi realizado em um fragmento de Floresta Ombrófila Mista Submontana (IBGE, 2012), com área aproximada de 103,06 ha e altitude que varia de 990 a $1000 \mathrm{~m}$, localizado na latitude 27 51'19.20"S e longitude 50¹0'33,39”'W, no município de Lages, Santa Catarina. De acordo com Higuchi et al. (2013), áreas de Floreta Ombrófila Mista na região apresentam elevado compartilhamento florístico com a Floresta Estacional Decidual, por não existir barreiras ecológicas e geográficas separando as mesmas. A área se encontra em avançado estágio de sucessão (SALAMI et al., 2014) e está integralmente protegida há, aproximadamente, 30 anos. O clima na região é considerado, segundo Köppen (1948), como $\mathrm{Cfb}$, temperado constantemente úmido, sem estação seca. A precipitação média anual é de $1.483 \mathrm{~mm}$, com chuvas bem distribuídas durante o ano e com uma temperatura média anual de $16,1^{\circ} \mathrm{C}$ (HIJMANS et al., 2004).

Para a caracterização dos atributos funcionais, foram consideradas as 20 espécies lenhosas mais abundantes a partir do trabalho de Higuchi et al. (2012), que estudaram o mesmo fragmento florestal. Estas espécies compõem a maioria dos indivíduos amostrados no trabalho, representando, assim, parte considerável da estrutura da floresta $(70,1 \%$ dos indivíduos lenhosos e $72,9 \%$ da área basal). Foram determinados, para cada espécie, os atributos funcionais de densidade média da madeira, tamanho médio das folhas, regime de renovação foliar, síndrome de dispersão e altura máxima no fragmento. Esses atributos, assim como suas respectivas importâncias em relação aos processos que influenciam nas características morfológicas, fisiológicas ou ecológicas das espécies arbóreas, foram definidos a partir de Van der Pijl (1982) e Cornelissen et al. (2003) (Tabela 1).

A densidade da madeira e o tamanho das folhas foram obtidos, de acordo com Pérez-Harguindeguy et al. (2013), a partir de indivíduos com CAP (circunferência a altura do peito, medida a 1,30 $\mathrm{m}$ do solo) igual ou superior a $15,7 \mathrm{~cm}$, pertencentes às 20 espécies pré-selecionadas, sendo estes indivíduos, 10 de cada espécie, obtidos de forma aleatória no fragmento. O levantamento dos dados de densidade da madeira foi realizado com coletas de amostras de madeira nos indivíduos amostrados de cada espécie com o uso de um trado de incremento de 5,15 mm, totalizando 200 amostras de madeira. Essas amostras, em laboratório, permaneceram imersas em água até a obtenção da massa úmida constante, que, por sua vez, foi obtido pelo método de deslocamento de água (princípio de Arquimedes), em que a medida da massa de água deslocada é igual ao volume da amostra de madeira imersa. Após, as amostras, foram mantidas em estufa para a estabilização da massa seca (corresponde a $0 \%$ de umidade). Após esse período de secagem, a obtenção da massa seca das amostras foi determinada pela pesagem em balança analítica. A determinação da densidade da madeira, então, foi realizada entre a razão da massa seca $(\mathrm{g})$ pelo volume $\left(\mathrm{cm}^{3}\right)$ de acordo com a norma ABNT NBR 11941-02 (2003).

A obtenção do tamanho da folha (área foliar) foi realizada a partir da coleta de 20 folhas em cada indivíduo. Com auxílio de um podão, foram coletadas folhas no terço mediano da copa, utilizando como critério a interceptação luminosa do dossel (folhas com incidência de luz), preferencialmente as sadias (CORNELISSEN et al., 2003). As amostras (folhas anexadas nos galhos) foram armazenadas em sacos plásticos fechados para evitar a perda de água até o manuseio das mesmas em laboratório. Posteriormente, em laboratório, as folhas foram fotografadas, com uma câmera conectada a um tripé com altura fixa, sendo 
TABELA 1: Atributos funcionais observados e suas respectivas importâncias em relação aos processos que influenciam no desenvolvimento das espécies arbóreas.

TABLE 1: Observed functional traits and their importance in relation to the processes that influence the development of tree species.

\begin{tabular}{|c|c|c|c|}
\hline Traço Funcional & Unidade & $\begin{array}{l}\text { Amostras } \\
\text { (inds./espécies) }\end{array}$ & Processos fisiológicos relacionados \\
\hline Densidade da madeira & $\mathrm{g} / \mathrm{cm}^{3}$ & 10 & $\begin{array}{l}\text { Longevidade, crescimento, altura máxima, } \\
\text { armazenamento de carbono. }\end{array}$ \\
\hline Tamanho da folha & $\mathrm{cm}^{2}$ & 10 & $\begin{array}{l}\text { Crescimento relativo, capacidade fotossintética, } \\
\text { longevidade, absorção de carbono. }\end{array}$ \\
\hline Renovação foliar & - & - & $\begin{array}{l}\text { Longevidade foliar, competição por luz, capacidade } \\
\text { fotossintética, eficiência do uso de nutrientes. }\end{array}$ \\
\hline Síndrome de dispersão & - & - & $\begin{array}{c}\text { Fecundidade, estruturação genética, regeneração, } \\
\text { sobrevivência. }\end{array}$ \\
\hline $\begin{array}{l}\text { Altura máxima no } \\
\text { fragmento }\end{array}$ & $\mathrm{m}$ & - & Competição por luz, crescimento, fecundidade. \\
\hline
\end{tabular}

a área foliar determinada por meio do programa ImageJ (RASBAND, 2007).

As informações referentes à determinação do regime de renovação foliar, síndromes de dispersão e altura máxima no fragmento foram obtidas por meio de observações de campo e revisão de literatura (LORENZI, 1998; 2000; CORNELISSEN et al., 2003; LOPES, 2010; SILVA et al., 2012).

Quanto ao regime de renovação foliar, as espécies foram classificadas como perenes ou decíduas; e quanto à síndrome de dispersão, as espécies foram classificadas de acordo com as classes propostas por Van Der Pijl (1982) em zoocóricas, anemocóricas e autocóricas.

Como alguns dos atributos analisados não são numéricos (renovação foliar e síndrome de dispersão), foi utilizada a distância de Gower (GOWER, 1971) para a construção de uma matriz de dissimilaridade entre as espécies, que posteriormente foi transformada em distância Euclidiana por meio do método de Cailliez (CAILLIEZ, 1983). Em seguida, as espécies foram agrupadas por meio de um dendrograma, com o algoritmo de Ward com método de ligação. Todas as análises foram realizadas no programa $\mathrm{R}$ (R CORE TEAM, 2014), utilizando as bibliotecas Vegan (OKSANEN et al., 2009) e FD (LALIBERTÉ; LEGENDRE, 2010; LALIBERTÉ; SHIPLEY, 2011).

\section{RESULTADOS E DISCUSSÃO}

Os resultados demonstraram que as espécies analisadas apresentam diferentes estratégias de investimento para alocação de recursos, como indicado pelos dados de densidade da madeira, tamanho médio da folha, regime de renovação foliar, síndromes de dispersão e altura máxima no fragmento (Tabela 2). Esses padrões podem ser o resultado das interações de competição por recursos, como, por exemplo, a luz, e as estratégias para sobrevivência durante os períodos desfavoráveis ao desenvolvimento, como no inverno, que na região é caracterizado por baixas temperaturas, cuja média da temperatura mínima para o mês mais frio no período de $1950-2000$ foi de $6,5^{\circ} \mathrm{C}$ (HIJMANS et al., 2004).

Entre as espécies de maior valor médio de densidade da madeira, destacaram-se aquelas pertencentes à família Myrtaceae e Salicaceae, que ocorreram predominantemente no estrato inferior da floresta e que foram classificadas como zoocóricas: Myrcia guianensis $\left(0,72 \mathrm{~g} / \mathrm{cm}^{3}\right)$, Banara tomentosa $\left(0,69 \mathrm{~g} / \mathrm{cm}^{3}\right)$, Casearia obliqua $\left(0,67 \mathrm{~g} / \mathrm{cm}^{3}\right)$, Calyptranthes concinna $\left(0,66 \mathrm{~g} / \mathrm{cm}^{3}\right)$ e Casearia decandra $\left(0,65 \mathrm{~g} / \mathrm{cm}^{3}\right)$. Já dentre as espécies com madeira de menor densidade, destacaram-se espécies do dossel superior com diferentes síndromes de dispersão: Sapium glandulosum $\left(0,32 \mathrm{~g} / \mathrm{cm}^{3}\right)$, Jacaranda puberula $\left(0,34 \mathrm{~g} / \mathrm{cm}^{3}\right)$ e Podocarpus lambertii $\left(0,43 \mathrm{~g} / \mathrm{cm}^{3}\right)$. Araucaria angustifolia, que é a espécie que melhor representa a fitofisionomia, apresentou densidade da madeira com valor intermediário $\left(0,5 \mathrm{~g} / \mathrm{cm}^{3}\right)$. 
TABELA 2: Características funcionais das 20 espécies arbóreas mais abundantes amostradas, ordenadas em ordem alfabética, em um fragmento de Floresta Ombrófila Mista Submontana em Lages - SC.

TABLE 2: Functional traits of the 20 most abundant tree species, sorted alphabetically, sampled in a Submontane Araucaria Forest fragment, in the municipality of Lages, SC state.

\begin{tabular}{|c|c|c|c|c|c|}
\hline Espécies & $\mathrm{D}$ & $\mathrm{TF}$ & $\mathrm{RF}$ & SD & $\mathrm{Hm}$ \\
\hline Allophylus edulis (A.St.-Hil. et al.) Hieron. ex Niederl. & 0,54 & 28,13 & $P$ & Zoocórica & 13 \\
\hline Araucaria angustifolia (Bertol.) Kuntze & 0,50 & 2,07 & $\mathrm{P}$ & Zoocórica & 26 \\
\hline Banara tomentosa Clos & 0,69 & 21,92 & $\mathrm{D}$ & Zoocórica & 13 \\
\hline Casearia decandra Jacq. & 0,65 & 9,04 & $\mathrm{D}$ & Zoocórica & 10 \\
\hline Casearia obliqua Spreng. & 0,67 & 26,16 & $\mathrm{D}$ & Zoocórica & 23 \\
\hline Calyptranthes concinna DC. & 0,66 & 12,04 & $\mathrm{P}$ & Zoocórica & 12 \\
\hline Cupania vernalis Cambess. & 0,57 & 298,03 & $\mathrm{P}$ & Zoocórica & 23 \\
\hline Dasyphyllum tomentosum (Spreng.) Cabrera & 0,56 & 58,72 & $\mathrm{D}$ & Anemocórica & 21 \\
\hline Duranta vestita Cham. & 0,46 & 12,19 & $\mathrm{D}$ & Zoocórica & 5 \\
\hline Jacaranda puberula Cham. & 0,34 & 212,87 & $\mathrm{D}$ & Anemocórica & 20 \\
\hline Lamanonia ternata Vell. & 0,51 & 99,05 & $\mathrm{D}$ & Anemocórica & 20 \\
\hline Lithraea brasiliensis Marchand & 0,64 & 17,02 & $\mathrm{P}$ & Zoocórica & 23 \\
\hline Matayba elaeagnoides Radlk. & 0,49 & 202,28 & $\mathrm{P}$ & Zoocórica & 23 \\
\hline Myrcia guianensis (Aubl.) DC. & 0,72 & 6,26 & $\mathrm{P}$ & Zoocórica & 13 \\
\hline Myrsine umbellata Mart. & 0,60 & 30,67 & $\mathrm{P}$ & Zoocórica & 19 \\
\hline Ocotea pulchella (Nees \&Mart.) Mez & 0,49 & 12,17 & $\mathrm{P}$ & Zoocórica & 25 \\
\hline Podocarpus lambertii Klotzsch ex Endl. & 0,43 & 3,67 & $\mathrm{P}$ & Zoocórica & 20 \\
\hline Sapium glandulosum (L.) Morong & 0,32 & 33,86 & $\mathrm{D}$ & Autocórica & 23 \\
\hline Zanthoxylum rhoifolium Lam. & 0,44 & 49,26 & $\mathrm{D}$ & Zoocórica & 19 \\
\hline Zanthoxylum kleinii (R.S.Cowan) P.G.Waterman & 0,58 & 27,52 & $\mathrm{D}$ & Zoocórica & 15 \\
\hline
\end{tabular}

Em que: $\mathrm{D}=$ densidade da madeira média $\left(\mathrm{g} / \mathrm{cm}^{3}\right) ; \mathrm{TF}=$ tamanho médio da folha $\left(\mathrm{cm}^{2}\right) ; \mathrm{RF}=$ regime de renovação foliar ( $\mathrm{P}=$ perenifólias, $\mathrm{D}=$ decíduas); $\mathrm{SD}=$ síndromes de dispersão; $\mathrm{Hm}=$ altura máxima no fragmento $(\mathrm{m})$.

Estes resultados podem ser parcialmente explicados pela existência de um trade-off clássico para o investimento de recursos em atributos funcionais que permitem o rápido crescimento em altura de espécies exigentes em luz e o que permitem maior capacidade de sobrevivência num ambiente sombreado de sub-bosque (KING et al., 2006). De fato, correlações negativas entre densidade da madeira e altura da árvore em ecossistemas florestais já foram relatadas na literatura (e.g. KOHYAMA et al., 2003). Destaca-se, também, que a variação na densidade da madeira representa um importante indicativo da capacidade de armazenamento de carbono, uma vez que o sequestro de carbono é um dos processos ecossistêmicos relacionado à densidade da madeira (CHAVE et al., 2009).

Araucaria angustifolia e Ocotea pulchella foram as árvores de maior porte, alcançando, respectivamente, até 26 e $25 \mathrm{~m}$ de altura, de forma que fazem parte do estrato emergente da floresta, destacando-se na fitofisionomia. Abaixo destas espécies, existe um conjunto de espécies com pelo menos $20 \mathrm{~m}$ de altura (Casearia obliqua, Cupania vernalis, Dasyphyllum tomentosum, Jacaranda puberula, Lamanonia ternata, Lithraea brasiliensis, Matayba elaeagnoides, Podocarpus lambertii e Sapium glandulosum), ocupando o dossel. Como espécies que ocorrem abaixo dos $20 \mathrm{~m}$, destacam-se as espécies Allophylus edulis, Banara tomentosa, Casearia decandra, Calyptranthes concinna, Duranta vestita, Myrcia guianensis, Myrsine umbellata, Zanthoxylum rhoifolium e Zanthoxylum kleinii. De acordo com Falser e Westoby (2005), a estrutura vertical de formações vegetacionais pode ser compreendida como sendo o resultado de uma "corrida" ecológica e evolucionária entre as diferentes espécies, competindo por 
luz. Assim, a distribuição ao longo do perfil vertical da floresta está relacionada com a partição do ambiente lumínico. Enquanto espécies no dossel têm acesso a 100\% da radiação fotossinteticamente ativa (RFA), as espécies no sub-bosque precisam apresentar estratégias para se desenvolverem com apenas uma fração desta. No entanto, dada a prioridade da alocação de seus recursos para investimento em altura ao invés de investimento em resistência e resiliência, espécies mais altas tendem a ser menos tolerantes a diferentes tipos de condições não favoráveis (MCGILL et al., 2006).

Além da competição por luz, destaca-se que o investimento em altura aumenta a capacidade de dispersão de sementes a distância (THOMSON et al., 2011), principalmente aquelas dispersas pelo vento, o que é um aspecto chave para a expansão espacial de populações e colonização de novas áreas (NATHAN et al., 2002). Se considerar, por exemplo, a dispersão anemocórica, a superfície de queda de propágulos vai depender da distância percorrida durante o transporte dos mesmos. Sendo assim, as espécies que apresentam dispersão anemocórica destacam-se em altura, ocorrendo predominantemente no dossel (GIEHL et al., 2007), como é o caso no presente estudo de Jacaranda puberula, Lamanonia ternata e Dasyphyllum tomentosum. No que se refere às síndromes de dispersão observadas, foi possível verificar resultados semelhantes em outros trabalhos realizados em Floresta Ombrófila Mista (ALMEIDA et al., 2008; NEGRINI et al., 2012; FERREIRA et al., 2013), na qual a zoocoria é considerada a estratégia de dispersão mais representativa.

Em relação às variações nos atributos foliares encontrados, ressalta-se a grande plasticidade fenotípica entre as espécies, como observado em estudos em regiões tropicais (ROZENDAL; HURTADO; POORTER, 2006). As espécies com os maiores valores de tamanho de folha foram Cupania vernalis $\left(298,03 \mathrm{~cm}^{2}\right)$, Jacaranda puberula $\left(212,87 \mathrm{~cm}^{2}\right)$ e Matayba elaeagnoides $(202,28$ $\mathrm{cm}^{2}$ ), todas com folhas compostas. As espécies com os menores valores foram as duas gimnospermas amostradas: Araucaria angustifolia $\left(2,07 \mathrm{~cm}^{2}\right)$ e Podocarpus lambertii $\left(3,67 \mathrm{~cm}^{2}\right)$. As variações existentes podem ser o resultado de adaptações ao longo do tempo a diferentes condições ambientais (REICH; WALTERS; ELLSWORTH, 1997; FONSECA et al., 2000; WRIGHT et al., 2004).

Com relação ao regime de renovação foliar, metade das espécies foi classificada como decíduas, sugerindo que a senescência de folhas representa uma importante estratégia ecológica no fragmento estudado. A deciduidade foliar tem sido relatada como uma adaptação para a sobrevivência durante períodos desfavoráveis do ponto de vista fisiológico, como, por exemplo, no inverno, em função da ocorrência de baixas temperaturas e geadas frequentes (CHABOT; HICKS, 1982). A coexistência de espécies perenes e deciduais em pequena escala espacial pode estar relacionada às variações nas condições edáficas (MURPHY; LUGO, 1986), uma vez que, em função do elevado "custo" da perda de biomassa por meio da queda de folhas, a deciduidade é uma estratégia que requer uma elevada disponibilidade de nutrientes nos solos (GIVNISH, 2002). A presença marcante de elementos florísticos deciduais pode ser explicada pelo fato de que na região Sul do Brasil a Floresta Ombrófila Mista e a Floresta Estacional Decidual compartilham um grande número de espécies (HIGUCHI et al., 2013). Para estes mesmos autores, isto se deve ao fato de as mesmas ocorrerem, em suas maiores extensões, nas mesmas bacias hidrográficas e da ausência de barreiras geográficas, favorecendo o compartilhamento de espécies.

A análise de agrupamento permitiu a identificação de dois grandes grupos de espécies, formados em função do regime de renovação foliar (Figura 1), com subdivisões subsequentes. O grupo das espécies perenes subdividiu-se em função do tamanho das folhas, sendo que o grupo das espécies de folhas grandes $\left(>200 \mathrm{~cm}^{2}\right)$ foi formado por Cupania vernalis e Matayba elaeagnoides e o de folhas menores $\left(<35 \mathrm{~cm}^{2}\right)$ foi formado por Podocarpus lambertii, Araucaria angustifolia, Ocotea pulchella, Lithraea brasiliensis, Myrsine umbellata, Allophylus edulis, Calyptranthes concinna e Myrcia guianensis. Já o grupo das espécies decíduas subdividiu-se em função da síndrome de dispersão, com espécies zoocóricas (Duranta vestita, Zanthoxylum rhoifolium, Banara tomentosa, Casearia decandra, Casearia obliqua e Zanthoxylum kleinii) e espécies anemocóricas e autocóricas (Sapium glandulosum, Lamanonia ternata, Dasyphyllum tomentosum e Jacaranda puberula).

O presente resultado reforça a importância do regime de renovação foliar como estratégia ecológica, de forma que os grupos funcionais foram definidos primeiramente em função dessa característica. Destaca-se que variações no regime foliar entre as espécies estão relacionadas aos diferentes mecanismos metabólicos (POORTER; BONGERS, 2006), o que reflete a existência de estratégias de vida distintas, fundamentais 


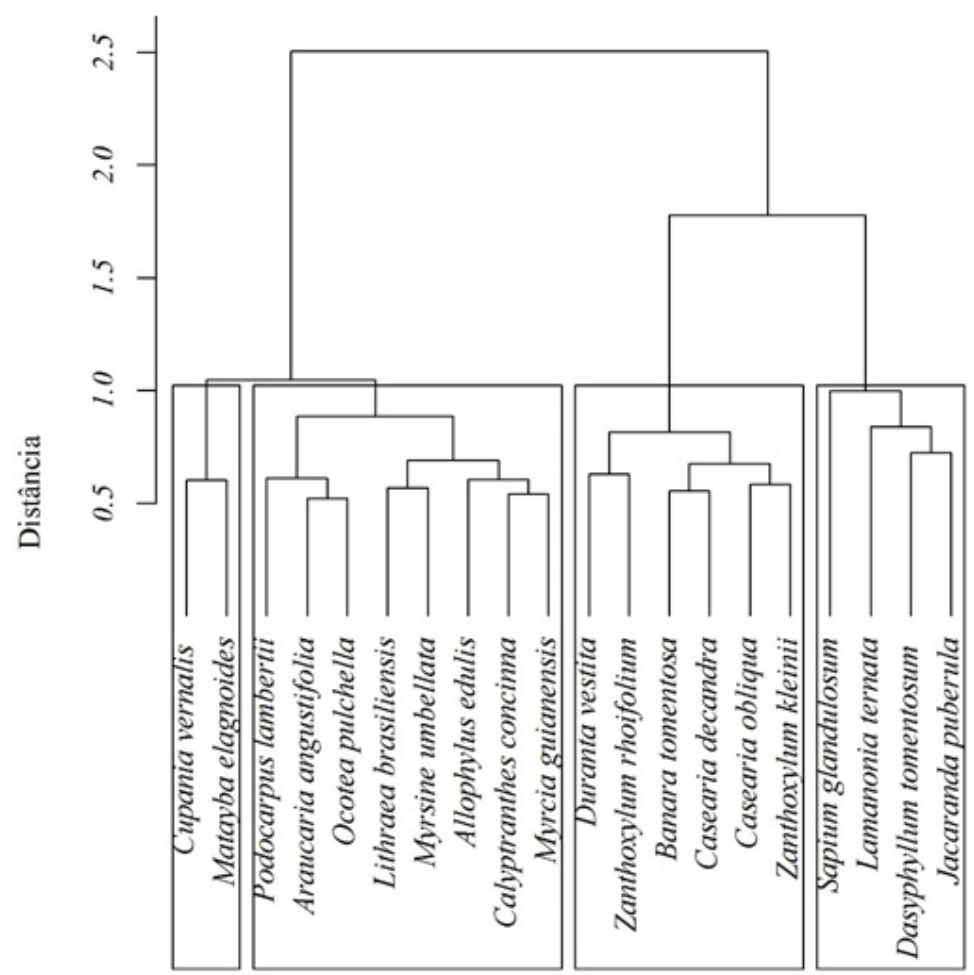

FIGURA 1: Dendrograma construído a partir dos atributos funcionais de 20 espécies arbóreas mais abundantes em um fragmento de Floresta Ombrófila Mista Submontana em Lages - SC. Grupo 1: Perenes de folhas maiores (Cupania vernalis e Matayba elaeagnoides); Grupo 2: Perenes de folhas menores (Podocarpus lambertii, Araucaria angustifolia, Ocotea pulchella, Lithraea brasiliensis, Myrsine umbellata, Allophylus edulis, Calyptranthes concinna e Myrcia guianensis), Grupo 3: Decíduas zoocóricas: (Duranta vestita, Zanthoxylum rhoifolium, Banara tomentosa, Casearia decandra, Casearia obliqua e Zanthoxylum kleinii) e Grupo 4: Decíduas não zoocóricas (Sapium glandulosum, Lamanonia ternata, Dasyphyllum tomentosum e Jacaranda puberula).

FIGURE 1: Dendrogram from functional traits of the 20 most abundant species in a Subontane Araucaria Forest Fragment, in the municipality of Lages, SC state. Group 1: Evergreen with large leaves (Cupania vernalis e Matayba elaeagnoides), Group 2: Evergreen with small leaves (Podocarpus lambertii, Araucaria angustifolia, Ocotea pulchella, Lithraea brasiliensis, Myrsine umbellata, Allophylus edulis, Calyptranthes concinna e Myrcia guianensis), Group 3: Deciduous with zoochoric syndrome (Duranta vestita, Zanthoxylum rhoifolium, Banara tomentosa, Casearia decandra, Casearia obliqua e Zanthoxylum kleinii), and Group 4: Deciduous with nonzoochoric syndrome (Sapium glandulosum, Lamanonia ternata, Dasyphyllum tomentosum and Jacaranda puberula).

para a partição de nichos ecológicos.

\section{CONCLUSÕES}

Conclui-se que as espécies mais abundantes do fragmento de Floresta Ombrófila Mista estudado, considerado em avançado estágio de sucessão, apresentaram variações dos atributos funcionais avaliados, sugerindo diferentes estratégias de vida. Foram identificados quatro grupos funcionais: 1) perenes de folhas grandes, 2) perenes de folhas pequenas, 3) decíduas zoocóricas e 4) decíduas não zoocóricas. Desta forma, infere-se que as diferenças funcionais entre os táxons avaliados sejam importantes para a organização 
florístico-estrutural do componente arbóreo, uma vez que estratégias de vida distintas são relevantes para a partição de nichos ecológicos em ecossistemas florestais.

\section{AGRADECIMENTOS}

À Universidade do Estado de Santa Catarina (UDESC) pela concessão de bolsa à primeira autora do trabalho, por meio do Programa de Bolsas de Monitoria de Pós-Graduação (PROMOP).

\section{REFERÊNCIAS}

ASSOCIAÇÃO BRASILEIRA DE NORMAS TÉCNICAS. NBR 11941-02: determinação da densidade básica em madeira. Rio de Janeiro, 2003. 6 p.

ALMEIDA, S. R. et al. Florística e síndromes de dispersão de um remanescente de Floresta Ombrófila Mista em sistema faxinal. Ambiência, Guarapuava, v. 4, n. 2, p. 289-297, 2008.

CAILLIEZ, F. The analytical solution of the additive constant problem. Psychometrika, Lisboa, v. 48, n. 2, p. 305-308, 1983.

CHABOT, B. F.; HICKS, D. T. The ecology of leaf life spans. Annual Review of Ecology and Systematics, Palo alto, v. 13, n. 1, p. 229-259, 1982.

CHAVE, J. et al. Regional and Phylogenetic Variation of Wood Density Across 2456 Neotropical Tree Species. Ecological Applications, Washington, v. 16, n. 6, p. 2356-2367, 2006.

CHABOT, B. F. et al. Towards a worldwide wood economics spectrum. Ecology Letters, Montpellier, v. 12, n. 4, p. 351-366, 2009.

CORNELISSEN, J. H. C. et al. Handbook of protocols for standardised and easy measurement of plant functional traits worldwide. Australian Journal of Botany, Melbourne, v. 51, n. 4, p. 335-380, 2003.

FALSTER, D. S.; WESTOBY, M. Alternative height strategies among 45 dicot rain forest species from tropical Queensland, Australia. Journal of Ecology, Oxford, v. 93, n. 3, p. 521-535, 2005.

FERREIRA, P. I. et al. Espécies potenciais para recuperação de Áreas de Preservação Permanente no Planalto Catarinense. Floresta e Ambiente, Rio de Janeiro, v. 20, n. 2, p. 173-182, 2013.

FONSECA, C. R. et al. Shifts in trait-combinations along rainfall and phosphorus gradients. Journal of Ecology, Oxford, v. 88, n. 6, p. 964-977, 2000.

GIEHL, E. L.H. et al. Espectro e distribuição vertical das estratégias de dispersão de diásporos do componente arbóreo em uma floresta estacional no sul do Brasil. Acta Botanica Brasílica, Feira de Santana, v. 21, n. 1, p. 137-145, 2007.

GITAY, H.; NOBLE, I. R. What are functional types and how should we seek them? In: SMITH, T. M.; SHUGART, H. H.; WOODWARD, F. I. Plant functional types: their relevance to ecosystem properties and global change. Cambridge: Cambridge University Press, 1997. p. 3-19.

GIVNISH, T. J. Comparative studies of leaf form: assessing the relative roles of selective pressures and phylogenetic constraints. New phytologist, Hoboken, v. 106, n. s1, p. 131-160, 1987.

GIVNISH, T. J Adaptive significance of evergreen vs. deciduous leaves: solving the triple paradox. Silva Fennica, Helsinki, v. 36, n. 3, p. 703-743, 2002.

GOWER, J. C. A general coefficient of similarity and some of its properties. Biometrics, Washington, v. 27 , n. 4 , p. $857-871,1971$.

HIGUCHI, P. et al. Influência de variáveis ambientais sobre o padrão estrutural e florístico do componente arbóreo, em um fragmento de Floresta Ombrófila Mista Montana em Lages, SC. Ciência Florestal, Santa Maria, v. 22, n. 1, p. 79-90, 2012.

HIGUCHI, P. et al. Influência do clima e rotas de migratórias de espécies arbóreas sobre o padrão fitogeográfico de florestas na região sul do Brasil. Ciência Florestal, Santa Maria, v. 23, n. 4, p. 539-553, 2013.

HIJMANS, R. J. et al. The WorldClim interpolated global terrestrial climate surfaces. Version 1.3. 2004. Disponível em: <http://www.worldclim.org>. Acesso em: 17 abr. 2014.

IBGE. Manual técnico da vegetação brasileira. 2. ed. Rio de Janeiro: IBGE, 2012. 272 p.

KING, D. A. et al. The role of wood density and stem support costs in the growth and mortality of tropical 
trees. Journal of Ecology, Oxford, v. 94, n. 3, p. 670-680, 2006.

KÖPPEN, W. Climatologia: con un estudio de los climas de la tierra. México: Fondo de Cultura Economica, 1948. $478 \mathrm{p}$.

KOHYAMA, T. et al. Tree species differentiation in growth, recruitment and allometry in relation to maximum height in a Bornean mixed dipterocarp forest. Journal of Ecology, Oxford, v. 91, n. 5, p. 797-806, 2003.

LALIBERTÉ, E.; LEGENDRE, P. A distance-based framework for measuring functional diversity from multiple traits. Ecology, Ithaca, v. 91, p. 299-305, 2010.

LALIBERTÉ, E; SHIPLEY, B. FD: measuring functional diversity from multiple traits, and other tools for functional ecology. R package version 1.0-11. [s. 1.: s. n.], 2011.

LAVOREL, S. et al. Plant functional classifications: from general groups to specific groups based on response to disturbance. Trends in Ecology and Evolution, Cambridge, v. 12, n. 12, p. 474-478, 1997.

LOPES S. F. Padrões florísticos e estruturais das florestas estacionais semideciduais do triângulo mineiro MG. 2010. 192 f. Tese (Doutorado em Ecologia) - Universidade Federal de Uberlândia, Uberlândia, 2010.

LORENZI, H. Árvores Brasileiras: manual de identificação e cultivo de plantas arbóreas nativas do Brasil. 2. ed. Nova Odessa: Instituto Plantarum, 1998. v. 1, 368 p.

LORENZI, H. Árvores Brasileiras: manual de identificação e cultivo de plantas arbóreas nativas do Brasil. 3. ed. Nova Odessa: Instituto Plantarum, 2000. v. 2, 352 p.

MATTOS, E. A. et al. Variação espacial e temporal em parâmetros fisioecoló-gicos de plantas. In: ROCHA, C. F. D.; ESTEVES, F. A.; SCARANO, F. R. Pesquisas de longa duração de Jurubatiba: ecologia, história natural e conservação. São Carlos: RiMa, 2004. p. 99-116.

MCGILL, B. J. et al. Rebuilding community ecology from functional traits. Trends in Ecology and Evolution, Cambridge, v. 21, n. 4, p. 178-185, 2006.

MURPHY, P. G.; LUGO, A. E. Ecology of tropical dry forest. Annual Review of Ecology and Systematics, Palo Alto, v. 17, n. 1, p. 67-88, 1986.

NATHAN, R. et al. Mechanisms of long-distance dispersal of seeds by wind. Nature, London, v. 418, n. 6896, p. 409-413, 2002.

NEGRINI, M. et al. Dispersão, distribuição espacial e estratificação vertical da comunidade arbórea em um fragmento florestal no planalto catarinense. Revista Árvore, Viçosa, v. 36, n. 5, p. 919-929, 2012.

OKSANEN, J. et al. Vegan: community ecology package. R package version. [s. 1. : s. n.], 2009. v. 1.

PÉREZ-HARGUINDEGUY, N. et al. New handbook for standardised measurement of plant functional traits worldwide. Australian Journal of Botany, Canberra, v. 61, n. 3, p. 167-234, 2013.

PILLAR, V. D.; DUARTE, L. D. S. A framework for metacommunity analysis of phylogenetic structure. Ecology Letters, Montpellier, v. 13, n. 5, p. 587-596, 2010.

POORTER, L.; BONGERS, F. Leaf traits are good predictors of plant performance across 53 rain forest species. Ecology, Ithaca, v. 87, n. 7, p. 1733-1743, 2006.

R CORE TEAM. R: A Language and Environment for Statistical Computing. Vienna: R Foundation for Statistical Computing, [2014]. Disponível em: <http://www.R-project.org>. Acesso em: 2 mar. 2014.

RASBAND, W. S. ImajeJ., version 1.42q. USA: National Institute of Health, 2007.

REICH P. B.; WALTERS, M. B.; ELLSWORTH, D. S. From tropics to tundra: global convergence in plant functioning. Proceedings of the National Academy of Sciences, Washington, v. 94, n. 4, p. 13730-13734, 1997.

ROZENDAL, D. M. A.; HURTADO, V. H; POORTER, L. Plasticity in leaf traits of 38 tropical tree species in response to light; relationships with light demand and adult stature. Functional Ecology, Oxford, v. 20, n. 2, p. 207-216, 2006.

SALAMI, B. et al. Influência de variáveis ambientais na dinâmica do componente arbóreo em um fragmento de Floresta Ombrófila Mista em Lages, SC The influence of environmental variables on tree species component dynamics in a montane araucaria forest fragment in Lages, SC. Scientia Forestalis, Piracicaba, v. 42, n. 102, p. 197-207, 2014.

SILVA, A. C. et al. Relações florísticas e fitossociologia de uma Floresta Ombrófila Mista Montana Secundária em Lages, Santa Catarina. Ciência Florestal, Santa Maria, v. 22, n. 1, p. 193-206, 2012. 
THOMSON, F. J. et al. Seed dispersal distance is more strongly correlated with plant height than with seed mass. Journal of Ecology, Oxford, v. 99, n. 6, p. 1299-1307, 2011.

VALLADARES, F.; BRITES, D. Leaf phyllotaxis: Does it really affect light capture? Plant Ecology, Dordrecht, v. 174, n. 1, p. 11-17, 2004.

VAN DER PIJL, L. Principles of dispersal in higher plants. Berlin: Springer-Verlag, 1982. 162 p.

VIOLLE, C. et al. Let the concept of trait be functional! Oikos, Copenhagem, v. 116, n. 5, p. 882-892, 2007. WALKER, B. H. Biodiversity and Ecological Redundancy. Conservation Biology, Cambridge, v. 6, n. 1, p. 18-23, 1992.

WEBB, C. T. et al. A structured and dynamic framework to advance traits-based theory and prediction in ecology. Ecology Letters, Montpellier, v. 13, n. 3, p. 267-283, 2010.

WESTOBY, M. et al. Plant Ecological Strategies: some leading dimensions of variation between species. Annual Review of Ecology and Systematics, Palo alto, v. 33, n. 1, p. 125-159, 2002.

WRIGHT, I. J. et al. The worldwide leaf economics spectrum. Nature, London, v. 428, n. 6985, p. 821-827, 2004. 